\title{
Frequent Traffic Flow Identification through Probabilistic Bloom Filter and its GPU-based Acceleration
}

\author{
Sisi Xiong ${ }^{\mathrm{a}, *}$, Yanjun $\mathrm{Yao}^{\mathrm{a}}$, Michael Berry ${ }^{\mathrm{a}}$, Hairong $\mathrm{Qi}^{\mathrm{a}}$, Qing Cao ${ }^{\mathrm{a}}$ \\ ${ }^{a}$ University of Tennessee, Knoxville, TN, USA
}

\begin{abstract}
Traffic measurement and monitoring are critical for many network applications, for example, bandwidth management and detecting security threats such as DoS (Denial of Service) attacks. In these cases, traffic is usually modeled as a collection of flows. One central problem is to identify those frequent flows, which account for a large percentage of total traffic, or whose frequency exceeds the user-specified threshold. In this paper, we describe a novel data structure called the probabilistic Bloom filter (PBF), which extends the classical Bloom filter into probabilistic direction, so that it can effectively estimate flows' frequencies, and identify frequent flows. We analyze the performance, tradeoffs, and capacity of this data structure, and investigate how to calibrate this data structure's parameters. We further develop one extension of the PBF for dynamic data stream needs. We implement our PBF on GPUs to gain more time efficiency. By testing with real network traces collected from a backbone router, we demonstrate that our method can keep track of flows' frequencies with adjustable accuracy, so that frequent flows can be identified with constant computational time complexity and low memory overhead.
\end{abstract}

Keywords: Network Measurement, Traffic Analysis, Bloom Filter.

\section{Introduction}

Accurate traffic monitoring and measurements are crucial in order to manage today's complex internet backbones, both for short-term purposes such as security needs

\footnotetext{
* Corresponding author

Email address: sxiong@vols.utk. edu (Sisi Xiong)
}

Preprint submitted to Journal of of Network and Computer Applications

January 21, 2017

(C) 2017. This manuscript version is made available under the Elsevier user license http://www.elsevier.com/open-access/userlicense/1.0/ 
(e.g., detecting traffic hot-spots, intrusions, and cyber-attacks) and long-term traffic engineering purposes with various QoS requirements [1, 2, 3, 4]. Frequent traffic flow identification has been a fundamental research topic in such applications by keeping track of all flows' frequencies based on real-time traffic flows. Given that the number of traffic flows between commercial end host pairs can be extremely large [1, 5], however, keeping a counter for each flow usually requires more memory than available on limited hardware resources, such as routers. Existing methods have addressed this problem through sampling and counting, such as NetFlow [6], where one of $N$ packets is sampled and counted. However, such methods can only sample a small portion of the entire traffic, leading to inaccurate results and over or under estimates.

Given such challenges, in this paper, we address the problem on efficiently identify15 ing those frequent traffic flows, whose frequency exceeds the user-specified threshold. To this end, we aim to build histograms of those frequent flows with limited memory space, so that we can easily identify whether a flow is "heavy" when it is encountered in the ongoing traffic. The key assumption in our approach is that, approximate knowledge on flows' frequency is already sufficient to identify heavy flow flows, as long as the frequency estimates can provide reliable upper and lower bounds associated with their most likely values.

In our previous work [7], we developed the probabilistic Bloom filter (PBF), which extends a highly memory compact probabilistic data structure, the classical Bloom filter, to support frequency queries. The key difference is that whenever a flow is inserted, instead of flipping the hash locations from 0 to 1 deterministically, we introduce a new parameter $p$, ranging from 0 to 1 , which represents the probability to flip the bit. Intuitively, more insertions of the same flow, i.e., a higher frequency of the flow, more bits will be flipped to 1 . Hence, the number of 1s is a reflection of flow's frequency. Based on this intuition, we derived an explicit formula to calculate the frequency based on the number of $1 \mathrm{~s}$.

However, our previous work left several problems open. First, there is no rigorous analysis on how to select the PBF's parameters, where a poorly selected set of parameters will cause PBF to generate inaccurate estimates. Second, the PBF was developed for handling static data, but real-world traffic datasets may usually exhibit streaming 
and dynamic nature [8]. Third, for large traffic datasets, PBF may lead to considerable computation overhead for calculating hash functions, which will have an impact on its time efficiency.

Therefore, in this paper, we extend our investigation to address these problems. In particular, we make the following contributions. First, we analyze our parameter 40 selection algorithm and make a key contribution in that we demonstrate that how parameter selection can be modeled by game theory, and we present an algorithm whose results lead to Nash equilibriums. Second, to process dynamic network traffic streams, we propose an enhanced version of the original PBF, called the time-decaying PBF (T-PBF), and demonstrate that it is more suitable for processing traffic streams. Third, 45 to accelerate processing of large traffic datasets, we develop a version of PBF supported by graphic processing units (GPUs), and verify its performance improvements by experiment comparisons. Finally, to allow PBF to be easily used, we also make an open-source python package of the PBF for other researchers and developers, which is available on Github [9].

To verify both PBF's and TPBF's effectiveness, we use three datasets of traffic traces collected from backbone routers to conduct experiments. According to result comparisons, our PBF can achieve better performance in accuracy and memory overhead compared to related approaches. Furthermore, our implementation on GPUs improves the time efficiency of the PBF further, compared to its CPU-based version, which makes the PBF a better choice when handling even large size of datasets.

The remainder of this paper is organized as follows: we survey related works in Section 2. The problem formulation and the PBF design are described in Section 3 . The extension of the PBF is presented in Section 4 . Next, we demonstrate that our parameter selection algorithm leads to Nash equilibrium in Section 5 and then we describe the implementation of the PBF on GPUs in Section 6 Finally the performance evaluation results are given in Section 7. We provide conclusions in Section 8 . 


\section{Related Work}

Identifying frequent flows in network traffic has been intensively studied in networking research community [10, 11]. The frequent flows are also referred as heavyhitters or iceberg flows [12], or elephant flows [3, 13], all of which frequency exceeds a certain threshold, or takes a certain fraction of all the traffic. A more general related problem is per-flow frequency estimation, which aims to yield frequency for each flow [14, 15]. Considering limited memory space and high traffic throughput on network devices, there are three main categories of solutions: one is to randomly sample all the packets, for example, random sampled netflow (RSN) [6]. However, such methods only sample a small portion of the entire traffic, leading to inaccurate results and cannot preserve integrated flow states. Another category is counter-based algorithms, for example, frequent algorithm [16, 17], lossy counting [18] and space-saving algorithm [19]. These methods decrease memory usage through keeping counters only for frequent flows, and they don't support deletions, hence they are not applicable in fully dynamic data streams. The last category is sketch-base algorithms, for example, count sketch [20], count-min sketch [21], group testing [22] and hCount [23]. Sketch based algorithms support per-flow frequency estimation without storing flow identifiers explicitly, and they support deletions, hence they are the only alternatives in dynamic data streams [11].

The Bloom filter (BF) [24] is a space-efficient randomized data structure that answers the question on whether an element is in a set. See Section 3.2 for a brief introduction. Due to its bit nature, Bloom filter is highly compact in memory space, with the sacrifice of predictable false positive error rates. However, false negative never 85 happens.

In its initial design, Bloom filter did not address the issues of element duplicates. Counting Bloom filters (CBFs) [13, 25, 26, 27] have been designed to address this issue. They adopted fixed size counters (also called bins) instead of single bits in Bloom filter's vector design. When an item is inserted, the corresponding counters are increased, hence, duplicate information is maintained. Therefore, CBFs require memory overhead that is usually an order of magnitude higher than common BFs. 
To support skewed distributions in data streams using CBF, Cheng and others [28] introduced the decaying operation by decreasing the counters periodically. In another approach, Kumar and others [29] proposed the space-code Bloom filter (SCBF) (later extended to a multi-resolution version called the MRSCBF), which used a filter made up of a fixed number of groups of hash functions. During the insertion operation, one group of hash functions was randomly chosen for the element. For query, the number of groups containing the element was counted to estimate the frequency. However, as only open formulas were provided, estimating frequency was done by looking up a pre-computed table, which was very computationally intensive to be built. Liu and others [30] proposed the reversible multilayer hashed counting Bloom filter (RML$\mathrm{HCBF}$ ), whose hash functions select a set of consecutive bits from the original strings as hash values, so that it may find elephant flows (large and continuous flow) using the counter values and thresholds. Bai and others [31] proposed an enhaced version of the multistage Bloom filters by introducing a largeflow-CBF and dynamic counters. Zhang and others [32] adapted the Bloom filters and LRU to keeps track of the frequent flows, while ignoring those are not. Such efforts differ in our work, since there are no counters or multiple data structures in our design, yet we can still yield frequency estimation results. To this end, we present closed-form results on modeling the performance of the proposed data structures.

In terms of GPU-based acceleration, Costa [33] proposed the BloomGPU, which is a library that supports two operations, insert and query, of Bloom filter on GPUs. Shi and others [34] adopted GPU-based Bloom filter to accelerate error correction in DNA-sequence data. Moreover, Ma and others [35] utilized shared memory as an optimization, and theoretically analyzed how to assign GPU architecture parameters according to the Bloom filter's parameters. However, all these works were developed for original Bloom filter, which is different from our PBF. Therefore, we make different design choices when we implement PBFs on GPUs. 


\section{Probabilistic Bloom Filter}

120 present its programming interfaces, followed by the operations between multiple PBFs, and finally, the analysis of its properties, capacity and performance.

\subsection{Problem Formulation}

We first present the problem formulation: given a set of $n$ network packets $P=$ identified by source or destination addresses, or source-destination address pair, or the five-tuple identity (source-destination addresses, source-destination port numbers, and protocol identifier), depending on user's specification. Suppose there are $N$ flows $F=\left\{f_{1}, f_{2}, \ldots, f_{N}\right\}$, the problem of identifying frequent flows is defined as finding

\subsection{Bloom Filter}

A Bloom filter is an array of $m$ bits that are initialized to all $0 \mathrm{~s}$, and its functionality is to identify membership, i.e., whether an element is in a set. The Bloom filter supports two basic operations: insertion and query. By inserting an element, $k$ hashed positions, which are calculated based on the element, out of $m$ bits are set to 1 . By querying an element, $k$ hashed positions are tested against 1 , and any 0 findings indicate that the element is not in the set. There are two types of errors: false negative errors never happen to Bloom filter, i.e., an element that is actually in the set is always returned as it is. However, false positive errors could happen with a probability, meaning that an element is not in the set might be reported as it is, since the $k$ bits in the Bloom filter might be set to 1 by other elements due to hash collisions. The false positive error probability $p$ relates to the size (length) of the Bloom filter $m$, the number of elements inserted $n$, and the number of hash functions $k$, according to

$$
p=\left(1-e^{-k n / m}\right)^{k} .
$$




\subsection{Programming Interfaces of $P B F$}

145

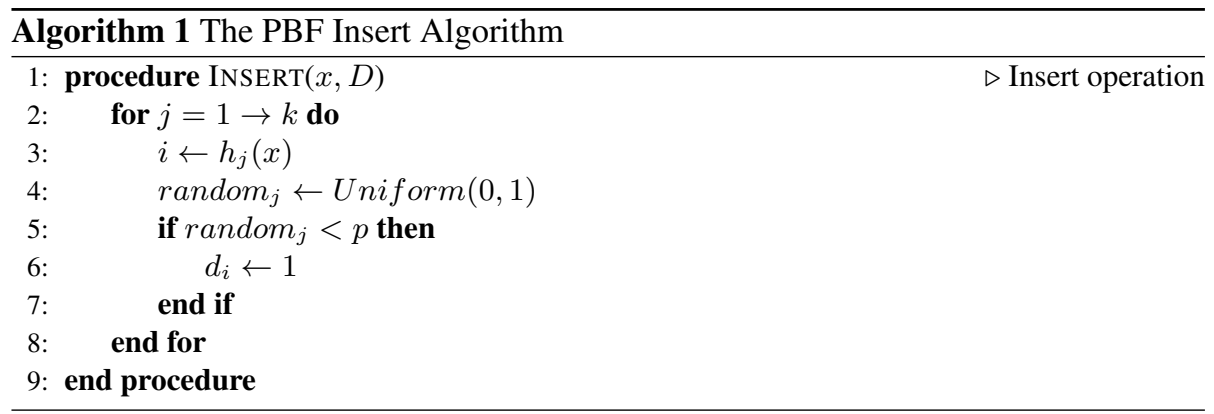

We first describe the INSERT operation, whose details are illustrated in Algorithm 1. For this operation, the primary change compared to a conventional Bloom filter is that it uses a parameter $p$ to decide whether to flip a bit from 0 to 1 , when items are inserted. For example, as shown in Figure 1 , we choose $m=25, k=3, p=2 / 3$, when inserting an item $e_{1}$, only two bits are flipped due to the new parameter $p$. Note that as an optimization, we do not need to read the bit's value before we set it to 1 , thereby reducing the number of memory accesses in the insert operation.

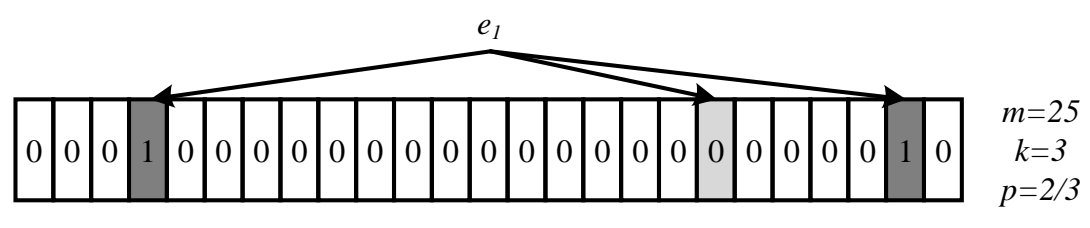

PBF vector

Figure 1: Insert operation of PBF. 
For the frequency query algorithm, it will simply add up the number of $1 \mathrm{~s}$ in the $k$ bits as determined by the hash functions, and uses statistical inference methods to obtain an approximate frequency of the data item in the dataset. We will describe the details of this estimation operation in Section 3.4

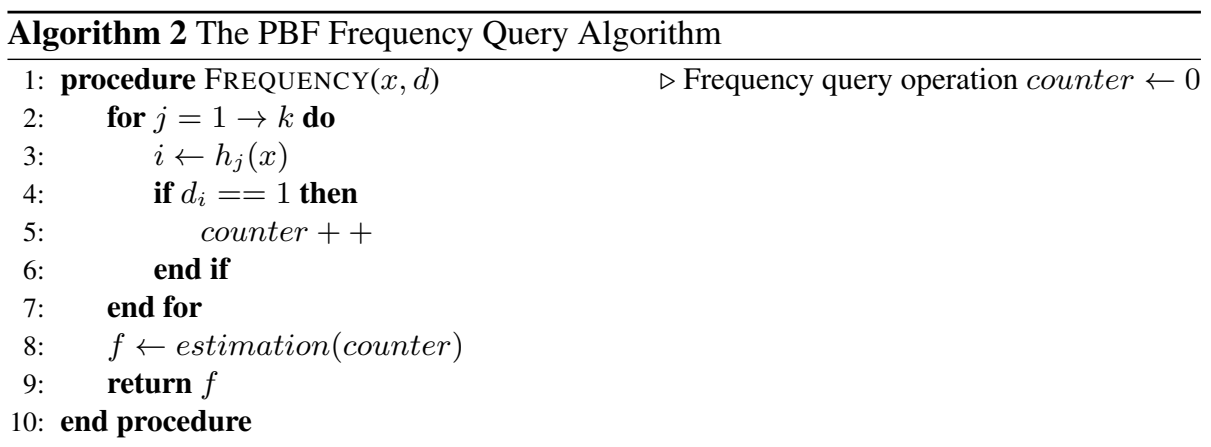

For multiple PBFs, we provide two APIs, JOIN and COMPRESS, which are facilitated by the bit-vector nature of PBFs. Given two multi-sets $S_{1}$ and $S_{2}$, suppose that they are represented by two PBFs, $D 1$ and $D 2$, we can calculate the PBF that represents the union set $S=S_{1} \cup S_{2}$ by taking the OR of their PBFs, $D=D 1 \vee D 2$, assuming that the bit vector length $m$ and the hash functions are identical. The merged filter $D$ represents the aggregate frequency of an item belonging to $S_{1}$ or $S_{2}$ as belonging to the set $S$.

The second operation is compression. If the PBF size $m$ is divisible by 2 , the compression operation allows us to store the original multiset in a shorter bit vector. This can be achieved by bit-wise OR of the first and second halves of the PBF's bit vector together. To insert or query the new PBF, the range of the hashing functions also needs to be updated by applying the $\bmod (\mathrm{m} / 2)$ operation to their outputs.

\subsection{Performance Modeling of PBFs}

Because the PBF introduces one additional parameter $p$, it has different properties compared to the original BF. In our previous paper, we model the performance of PBF by studying relationship between frequency of items and number of bits that are flipped out of $k$ hashed positions. Table 1 shows all the notations. We derive the explicit 


\begin{tabular}{|l|l|}
\hline$f$ & The frequency threshold of heavy-hitter items \\
\hline$k$ & The number of hashing functions \\
\hline$m$ & The length of the bit-vector \\
\hline$n$ & The total number of packets or items in a dataset \\
\hline$p$ & The probability for setting a bit to 1 \\
\hline$\hat{y}$ & The observed number of 1 s in the bit-vector \\
\hline
\end{tabular}

formula to calculate estimated frequency $f$ with respect to number 1 s reserved $\hat{y}$ and

PBF's parameters, according to

$$
f=\frac{k n p+m \ln \left[1-\frac{\hat{y}}{k}\right]}{(k-m) p} .
$$

Next, we calculate the confidence interval for $f$, by approximating it using a normal distribution based on the central limit theorem. This is also the so-called Wald Method, and we can derive the lower and upper bounds for $f$ as shown below [7]:

$$
\begin{aligned}
& f_{\text {min }}=\frac{k n p+m \ln \left[\frac{k-\hat{y}}{k}+\sqrt{\frac{\left(1-\frac{k-\hat{y}}{k}\right)(k-\hat{y})}{k^{2}}} z_{\frac{1}{2} \alpha}\right]}{(k-m) p} \\
& f_{\text {max }}=\frac{k n p+m \ln \left[\frac{k-\hat{y}}{k}-\sqrt{\frac{\left(1-\frac{k-\hat{y}}{k}\right)(k-\hat{y})}{k^{2}}} z_{\frac{1}{2} \alpha}\right]}{(k-m) p}
\end{aligned}
$$

As an illustrative example, suppose we want to filter data traffic with $100 \mathrm{~K}$ flows, where frequent flows are defined as those with a frequency to be at least one percent of the total, i.e., $1 K$ flows. We pick a Bloom filter size of $m=2 M$ bits $\left(1 M=10^{6}\right)$, and let $k=1000$. We select $p$ as 0.0006 (we will explain this later in parameter selection). In this case, the frequent flow is expected to have 467 bits in 1000 bits set as 1 . Conversely, if indeed, 467 bits are set, the estimated number of flows is 999 , with a $95 \%$ confidence interval as $[905,1098]$. On the other hand, if somehow the value of $\mu-2 \sigma=435$ bits are set, the number of estimated flows is 902 , with a $95 \%$ confidence interval as $[813,995]$. Note that, however, this interval does not contain 1000 as it is a $95 \%$ confidence interval. One may want to use the $99 \%$ confidence interval to capture 


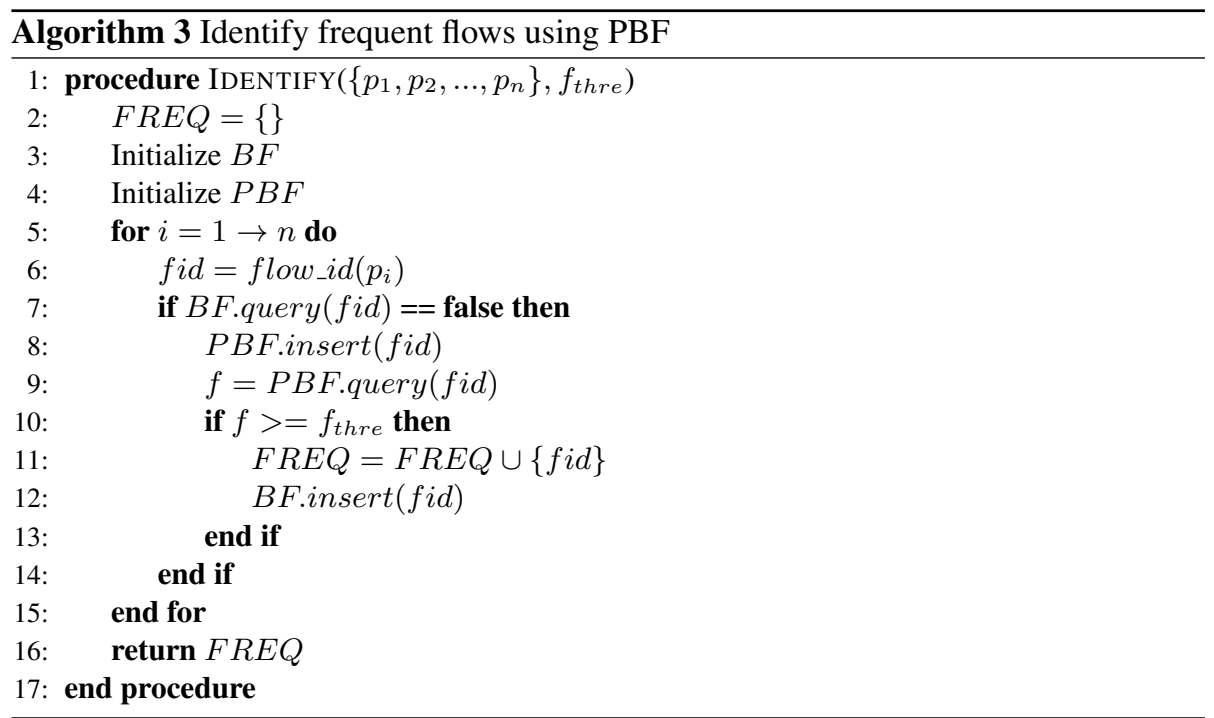

a larger range. In this case, the $99 \%$ interval gives the bound as $[797,1013]$, which indeed contains the 1000 in its range.

\subsection{Identifying frequent flows and flow frequency estimation using PBF}

Depending on user's specification and parameter settings, the PBF can be configured as a tool of identifying frequent flows, or a tool of per-flow frequency estimation. For frequent flow identification, the procedure using the PBF works as Algorithm 3 describes, where we use a conventional Bloom filter $B F$ to avoid duplicate queries on frequent flows. When a packet arrives, we first query its flow identifier using $B F$. If the flow identifier is in $B F$, we consider that the packet belongs to a frequent flow. If not, we insert the flow identifier into a $P B F$, and estimate its frequency. If it exceeds the threshold, we consider it as a frequent flow and we insert the flow identifier into $B F$.

According to analysis in our previous paper [7], through carefully parameter tuning, the PBF can also configured as a per-flow frequency estimation. However, since the PBF is a bit-array and it does not store flow identifiers explicitly, we also use a conventional Bloom filter to filter out duplicate flow identifiers, and a seperate flow identifier table for further frequency query operation. After inserting flow identifiers of 


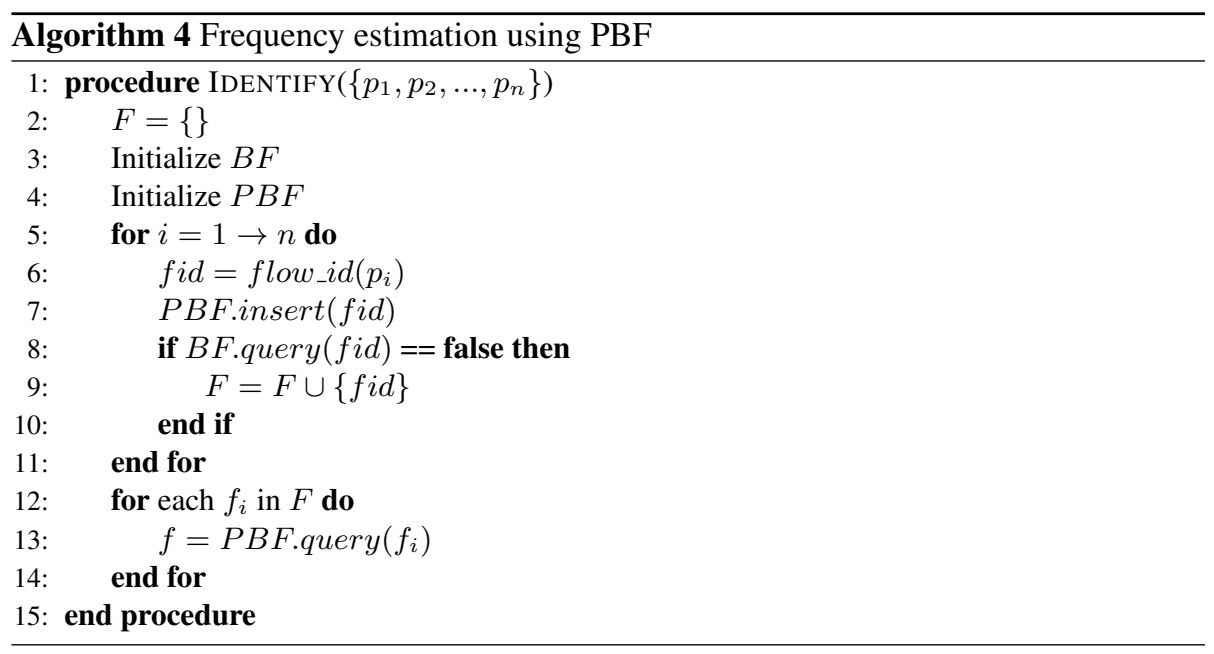

all packets into PBF, we query the PBF using all the items in the flow identifier table to conduct frequency estimation. The algorithm is shown in Algorithm 4

\section{Extension of the PBF}

To further expand the ability of PBF, we have developed one extension, a timedecaying PBF (T-PBF). The key motivation for T-PBF is that in some scenarios, large stream data would saturated the PBF vector as data continues to accumulate and the accuracy would be affected for long term estimation. Therefore, we propose the T$\mathrm{PBF}$, which allows it to forget those frequent items that appeared in the distant past.

\subsection{T-PBF: Time-decaying PBF Design}

The idea of the T-PBF is to introduce a new operation, decaying, which flips bits from 1 to 0 with a probability $q$ over each time epoch $T$. This can be considered as an approximation for the delete operation. Formally, this updated algorithm is shown in Algorithm 5

We now demonstrate the long term behavior of the T-PBF. For simplicity, we consider operations in epochs, and the decaying operation only occurs at the end of each epoch. Observe that for each bit, for each epoch, it may either start with bit 1 or 0 . If it starts with 1 , it may be flipped to 0 at the end of the epoch with a probability of 


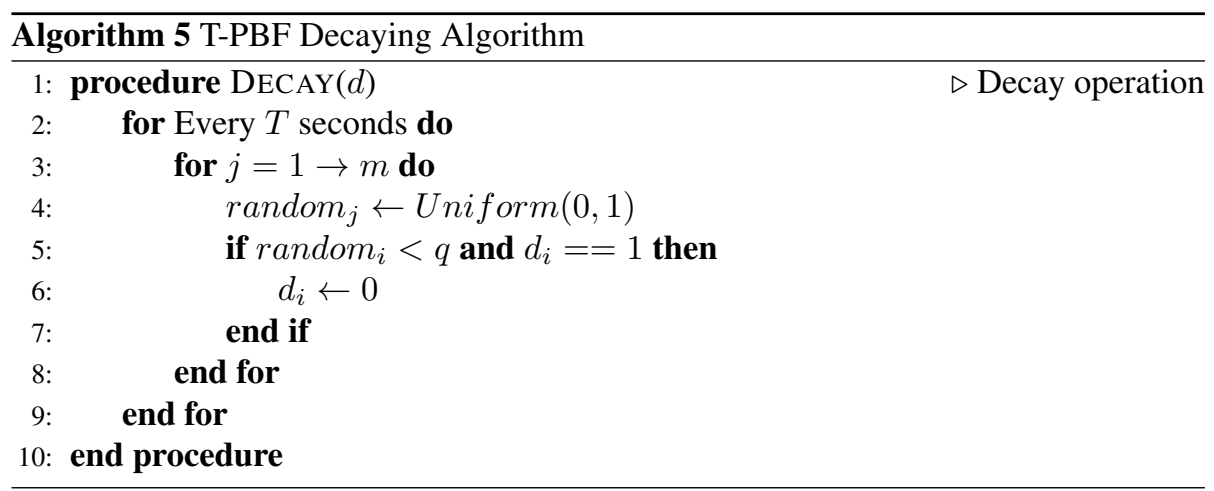

q. However, if it starts with 0 , it may be flipped to 1 first, and then flipped back to 0 with a probability of $q$. Therefore, we can use a discrete time Markov chain to describe these operations. In particular, because the transitions exhibit different probabilities at the beginning and the end of each epoch, we can model it with a time-inhomogeneous chain with a seasonal variation. In this case, we have that the seasonal period $d=2$, and the transition probability as the following (assuming $n \geq 0$, while $2 n$ and $2 n+1$ stand for the index of epochs)

$$
P(2 n)={ }_{1}^{0}\left[\begin{array}{cc}
0 & 1 \\
1-\alpha & \alpha \\
0 & 1
\end{array}\right], \quad P(2 n+1)={ }_{1}^{0}\left[\begin{array}{cc}
0 & 1 \\
1 & 0 \\
\beta & 1-\beta
\end{array}\right],
$$

where we have:

$$
\begin{array}{ll}
\alpha= & 1-e^{-\frac{p k(n-f)}{m}} \times e^{-p f}, \text { and } \\
\beta= & q .
\end{array}
$$

To analyze this seasonal chain, we can add a supplementary variable to create a homogeneous chain. The new chain contains four states: $A(0, a), B(0, b), C(1, a), D(1, b)$. Its transition matrix is shown below, followed by the corresponding Markov chain illustration. 


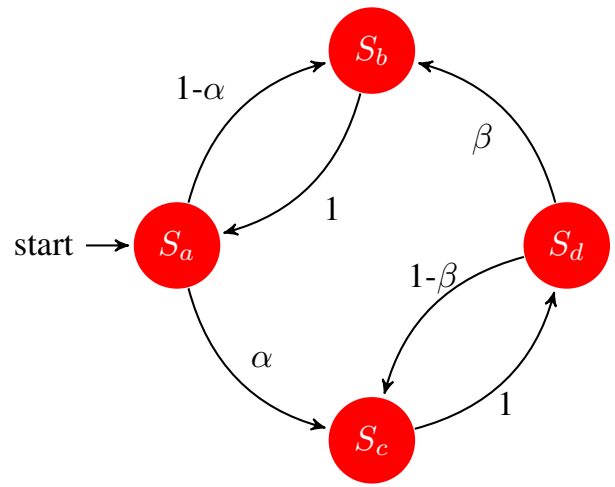

Figure 2: The Markov Transition for the T-PBF.

$$
P(n)=\begin{gathered}
A \\
B \\
C \\
D
\end{gathered}\left[\begin{array}{cccc}
0 & 1-\alpha & \alpha & 0 \\
1 & 0 & 0 & 0 \\
0 & 0 & 0 & 1 \\
0 & \beta & 1-\beta & 0
\end{array}\right]
$$

This Markov chain has four communicating classes, $A, B, C$, and $D$. They are all recurrent classes, as well. In the long term, this chain has the following stationary distribution:

$$
P(s)= \begin{cases}\frac{\beta}{2 \alpha+2 \beta} & \text { for } s \equiv A, \\ \frac{\beta}{2 \alpha+2 \beta} & \text { for } s \equiv B, \\ \frac{\alpha}{2 \alpha+2 \beta} & \text { for } s \equiv C, \\ \frac{\alpha}{2 \alpha+2 \beta} & \text { for } s \equiv D .\end{cases}
$$

As expected, the results show that in the long term, if a flow is no longer available, $f$ will decrease to 0 , and its $\alpha$ will quickly converge to $1-e^{-\frac{p k n}{m}}$, which is smaller.

Therefore, in the long run, most bits will be reset to 0 , as demonstrated by the increased probability of states $A$ and $B$ in Figure 2. This validates the design of the time-decaying nature of the T-PBF. On the other hand, if a flow re-appears with a large $f$, its transition will mostly stay in states $C$ and $D$, which means that more bits will be set due to the 
flow existence. Note that the epoch of the T-PBF can also be dynamic: as the PBF will lose the ability of prediction when all $k$ bits are set to 1 s, when a large percentage of $k$ bits for any element are set to 1 , the decaying process can be triggered.

\subsection{Selection of Parameters for T-PBF}

To perform accurate frequency estimations with T-PBF, it is critical to choose proper values for parameters, such as $m, k, p$, and $q$. As T-PBF extends PBF by introducing one additional parameter $q$, and the decaying operations are only triggered at the end of each period, we can use the same algorithms to choose the values of $m$, $k$, and $p$, based on what we discussed in our previous paper [7]. As a result, in the following part, we will discuss how to choose the value for $q$.

For an element, at the end of the first epoch, the probability of a bit is still 1 is

$$
\theta_{1}^{\prime}=\theta_{1} *(1-q),
$$

where $\theta_{1}$ is the probability that a bit is set to 1 during each epoch.

Then at the end of the second epoch, the probability of a bit is 1 would be a joint effect of the first two epoch, therefore

$$
\theta_{2}^{\prime}=\left(\theta_{2}+\theta_{1}^{\prime}-\theta_{2} * \theta_{1}^{\prime}\right) *(1-q)
$$

Finally, at the end of the ep-th epoch, the probability of a bit is 1 would be

$$
\theta_{e p}^{\prime}=\left(\theta_{e p}+\sum_{i=1}^{e p-1} \theta_{i}^{\prime}-\theta_{e p} \prod_{i=1}^{e p-1} \theta_{i}^{\prime}\right) *(1-q),
$$

as in long term, $\theta_{e p} \prod_{i=1}^{e p-1} \theta_{i}^{\prime} \rightarrow 0$, we can have the estimate probability as

$$
\theta_{e p}^{\prime} \approx \sum_{i=1}^{e p} \theta_{i}(1-q)^{e p-i}
$$

To guarantee the prediction accuracy, we need to prevent over-saturating the $k$ bits for any element. Therefore, we need to make sure that

$$
\forall \sum_{i=1}^{e p} y_{i}(1-q)^{e p-i} \leq k(1-\epsilon),
$$


where $e p$ is the number of passing epochs, $\epsilon$ is a threshold with a small value, and $y_{i}$ is the number of bits set to $1 \mathrm{~s}$ with the insertion of an element in the $i$ th epoch. As a result of above requirements, we can rewrite this equation as

$$
\max \sum_{i=1}^{e p} y_{i}^{\prime}(1-q)^{e p-i} \leq k(1-\epsilon),
$$

where $y_{i}^{\prime}$ is the number of 1 generated by the most frequent element to the bit array. By setting $y_{i}^{\prime}=y_{\max }=\max _{i=1}^{e p} \max _{j=1}^{t} y_{i j}$, which is the maximum number of $1 \mathrm{~s}$ set by the element with the largest frequency in all epochs, so that $t$ is the number of elements in each epoch, then

$$
\sum_{i=1}^{e p} y_{\max }(1-q)^{e p-i} \leq k(1-\epsilon),
$$

then

$$
\left(1-e^{-\frac{p k(n-f \max )}{m}} e^{-p f_{\max }}\right) \frac{1-(1-q)^{e p}}{q} \leq 1-\epsilon .
$$

For a long run, where $(1-q)^{e p} \rightarrow 0$, then we have:

$$
\frac{1-e^{-\frac{p k\left(n-f_{\max }\right)}{m}} e^{-p f_{\max }}}{1-\epsilon} \leq q \leq 1 .
$$

\section{PBF Parameter Selections are Nash Equilibrium}

As discussed in our previous paper [7], the estimation accuracy of the PBF and the T-PBF would be poor if an improper combination of parameters is chosen and used. In the following, we demonstrate that our algorithms for parameter selection will always lead to Nash equilibriums in practice, i.e., further adjusting any parameter by itself will not lead to a better performance [36]. We do not, however, claim that the parameter selection is globally optimal, as we find such optimality may depend on the particular application requirements. Indeed, the tradeoffs between the parameters are complex, and optimizing for any single global metric out of context is not particularly meaningful. Our proof is based on modeling the rules for parameters as the strategies 
in a non-cooperative game. Specifically, we prove that:

Theorem 5.1. The parameter selection algorithm for the PBF outputs a set of parameters that forms a Nash equilibrium.

Proof To prove that the PBF parameter selection algorithm leads to Nash equilibriums, we define a normal form game as a triple $\left(N,\left(S_{i}\right)_{i \in N},\left(c_{i}\right)_{i \in N}\right)$, where

- $N$ is the set of players, and $N=3$;

- $S_{i}$ is the set of strategies of the player $i$. Each player can choose to follow or not to follow a specific rule for a parameter $(p, m$, or $k$ ), as in our previous paper [7], where $S_{1}=\{$ Follow_P,Not_Follow_P $\}, S_{2}=\left\{F o l l o w \_K, N o t \_F o l l o w \_K\right\}$, and $S_{3}=\left\{\right.$ Follow_M $_{-}$Not_Follow_M $\}$;

- $S=S_{1} \times S_{2} \times S_{3}$ is the set of states;

- a state is $s=\left(s_{1}, \ldots, s_{3}\right) \in S$;

- $c_{i}: S \rightarrow \Re$ is the cost function of player $i \in N$. In the state $s$ player $i$ has a cost of $c_{i}(s)$. The cost is defined as the difference between the upper and lower estimation bounds, which represents the estimation accuracy.

In general, the cost value is computed as (based on results from 3 and 4 ):

$$
C(p, k, m)=f_{\max }-f_{\min }=\frac{m(\ln \epsilon-\ln (1-\epsilon))}{(k-m) p} .
$$

Based on the Nash Theorem [36], which claims that every finite normal form game has a mixed Nash equilibrium, the PBF parameter selection game has a mixed Nash equilibrium. In that case, in the following part, we will show that the state as generated in the PBF parameter selection algorithm forms one Nash equilibrium for the game.

As described in the game, each player has two strategies as following or not following the rule for a specific parameter. For player 1, the cost for the game is as shown in Table 2 
Table 2: Cost for player 1.

\begin{tabular}{|l|l|l|l|l|}
\hline & $($ FK,FM $)$ & (NFK,FM $)$ & (FK,NM) & (NK,NM) \\
\hline FP & C(p, k, m) & C(p, k', m) & C(p, k, m') & C(p, k', m') \\
\hline NFP & C(p', k, m) & C(p', k', m) & C(p', k, m') & C(p', k', m') \\
\hline
\end{tabular}

Table 3: Cost for player 2.

\begin{tabular}{|l|l|l|l|l|}
\hline & (FP,FM $)$ & (NFP,FM) & (FP,NFM $)$ & (NFP,NFM) \\
\hline FK & $\mathrm{C}(\mathrm{p}, \mathrm{k}, \mathrm{m})$ & $\mathrm{C}(\mathrm{p}, \mathrm{k}, \mathrm{m})$ & $\mathrm{C}\left(\mathrm{p}, \mathrm{k}, \mathrm{m}^{\prime}\right)$ & $\mathrm{C}\left(\mathrm{p}, \mathrm{k}^{\prime}, \mathrm{m}^{\prime}\right)$ \\
\hline NFK & $\mathrm{C}(\mathrm{p}, \mathrm{k}, \mathrm{m})$ & $\mathrm{C}\left(\mathrm{p}^{\prime}, \mathrm{k}^{\prime}, \mathrm{m}\right)$ & $\mathrm{C}\left(\mathrm{p}, \mathrm{k}^{\prime}, \mathrm{m}^{\prime}\right)$ & $\mathrm{C}\left(\mathrm{p}, \mathrm{k}^{\prime}, \mathrm{m}^{\prime}\right)$ \\
\hline
\end{tabular}
Other abbreviations follow the same format. Therefore, the cost for player 2 and player 3 are shown in Table 3 and Table 4

Now, we will find the best response strategy of the three players. Recall that the parameter selections can be summarized as $-\frac{\log (1-\epsilon)}{n} \leq p \leq \frac{(n-f) \log (1-\epsilon)-n \log (\epsilon)}{n f}$, $k \geq 150$, and $m \geq \frac{-k n p}{\log (1-\epsilon)}$. Consequently, we can find the opposite of these strategies as $p^{\prime}<-\frac{\log (1-\epsilon)}{n}$ or $p^{\prime}>\frac{(n-f) \log (1-\epsilon)-n \log (\epsilon)}{n f}, k<150$, and $m<\frac{-k n p}{\log (1-\epsilon)}$, as $N F P, N F K$, and $N F M$, respectively. Let us start from player 1, whose costs for choosing different strategies are as follows:

$$
\begin{gathered}
C_{1}(F P)=C(p, k, m)+C\left(p, k^{\prime}, m\right)+C\left(p, k, m^{\prime}\right)+C\left(p, k^{\prime}, m^{\prime}\right), \\
C_{1}(N F P)=C\left(p^{\prime}, k, m\right)+C\left(p^{\prime}, k^{\prime}, m\right)+C\left(p^{\prime}, k, m^{\prime}\right)+C\left(p^{\prime}, k^{\prime}, m^{\prime}\right) .
\end{gathered}
$$

When the $k$ bits mapped by an element are saturated, we define the difference between upper and lower bounds of the estimated frequency as $\infty$, as the PBF can no longer provide an accurate estimation for the frequency. This corresponds to the case that we choose $p^{\prime}>$ $\frac{(n-f) \log (1-\epsilon)-n \log (\epsilon)}{n f}$, where we have $C_{1}(N F P)=\infty$, so $C_{1}(N F P)>C_{1}(F P)$. On the other hand, when $p^{\prime}<-\frac{\log (1-\epsilon)}{n}$, we also have that $C_{1}(N F P)>C_{1}(F P)$, as the cost is monotonically increasing with the decreasing of $p$. Hence, the best response strategy for player 1 is Follow_P.

Following the same procedure, the costs for player 2 under different strategies are:

$$
C_{1}(F K)=C(p, k, m)+C\left(p^{\prime}, k, m\right)+C\left(p, k, m^{\prime}\right)+C\left(p^{\prime}, k, m^{\prime}\right),
$$


Table 4: Cost for player 3.

\begin{tabular}{|l|l|l|l|l|}
\hline & (FP,FK) & (NFP,FK) & (FP,NFK) & (NFP,NFK) \\
\hline FM & C(p,k,m) & C(p',k,m) & C(p,k',m) & C(p',k',m) \\
\hline NFM & C(p,k,m') & C(p',k,m') & C(p,k',m') & C(p',k',m') \\
\hline
\end{tabular}

$$
C_{1}(N F K)=C\left(p, k^{\prime}, m\right)+C\left(p^{\prime}, k^{\prime}, m\right)+C\left(p, k^{\prime}, m^{\prime}\right)+C\left(p^{\prime}, k^{\prime}, m^{\prime}\right) .
$$

As the cost is monotonically increasing with the decreasing of $k$, and $k>k^{\prime}$, then we have that $C_{1}(F K)<C_{1}(N F K)$. In that case, the best response strategy for player 2 is Follow_K.

Now let us have a look at player 3 , whose costs under different strategies are as follows:

$$
\begin{gathered}
C_{1}(N F K)=C\left(p, k^{\prime}, m\right)+C\left(p^{\prime}, k^{\prime}, m\right)+C\left(p, k^{\prime}, m^{\prime}\right)+C\left(p^{\prime}, k^{\prime}, m^{\prime}\right) . \\
C_{1}(F M)=C(p, k, m)+C\left(p^{\prime}, k, m\right)+C\left(p, k^{\prime}, m\right)+C\left(p^{\prime}, k^{\prime}, m\right), \\
C_{1}(N F M)=C\left(p, k, m^{\prime}\right)+C\left(p^{\prime}, k, m^{\prime}\right)+C\left(p, k^{\prime}, m^{\prime}\right)+C\left(p^{\prime}, k^{\prime}, m^{\prime}\right) .
\end{gathered}
$$

As the cost function can be rewritten as $C(p, k, m)=\frac{\ln \epsilon-\ln (1-\epsilon)}{\frac{k p}{m}-p}$, we observe that its value will increase with the decrease of $m$. As $m>m^{\prime}$, we have that $C_{1}(N F M)>C_{1}(F M)$. In that case, the best response strategy is Follow_M for player 3 .

Above all, we have proven that $\left\{F_{\text {ollow_}} P, F_{\text {ollow_}} K, F_{\text {ollow_}} M\right\}$ is a Nash equilibrium for the game. In another word, the parameter selection algorithm of PBF is a Nash equilibrium.

Next, we consider the algorithm parameter selection for T-PBF, and we prove that:

Theorem 5.2. The parameter selection algorithm for the T-PBF with regard to $q$ generates a Nash equilibrium.

Proof The proof is similar to the previous proof, by analyzing the effects of $q$ to the strategies. The detailed proof is omitted due to space.

\section{Implementation of the PBF On GPUs}

According to the PBF operations, it uses more hash functions than regular Bloom 
hash functions calculations using heterogeneous hardware to achieve higher time efficiency. Our study is motivated by the observation that in recent years, general purpose GPUs (GPGPUs) have attracted significant interest due to their capacity to perform massive parallel operations. Therefore, we consider it would be ideal if we implement the PBF on GPUs, where we demonstrate that this is feasible using the NVIDIA CUDA platform.

\subsection{GPU Architecture Hierarchy}

We first give a brief description of GPU's architecture in the CUDA platform as the foundation of our design. Specifically, each GPU device contains one grid, where each item inside is referred to as a block. The blocks can be uniquely identified using either one, two or three numbers depending on the dimension of the grid. Within each block, there are individual threads that execute in parallel. Similar to the index of blocks, each thread can be identified by either one, two or three dimension numbers [37]. Based on this architecture design, the execution pattern of GPU is as follows: each block gets mapped to a streaming multiprocessor (SM), where the threads within the block are divided into groups called warps, which get executed using available CUDA cores. Generally, the warp size, i.e., the number of threads in each warp, is 32 for most current NVIDIA GPUs.

To fully utilize the computation power of GPUs, we mainly focus on two principles, which are guidelines of our PBF implementation. First, we try to assign the same amount of tasks to all threads in each warp, since the execution time of each warp is determined by the slowest thread in that warp. Second, to prevent memory bandwidth to become the bottleneck of our implementation, we try to make all threads in one warp either access the same memory addresses, due to the data broadcast function of GPUs, or access continuous data addresses.

\subsection{Implementation Overview}

In our implementation of PBF on GPUs, there are two components: the processing part which locates on GPU, usually referred as device in the literature on GPU processing, and the host program that is located on CPU side, as shown in Figure 3 


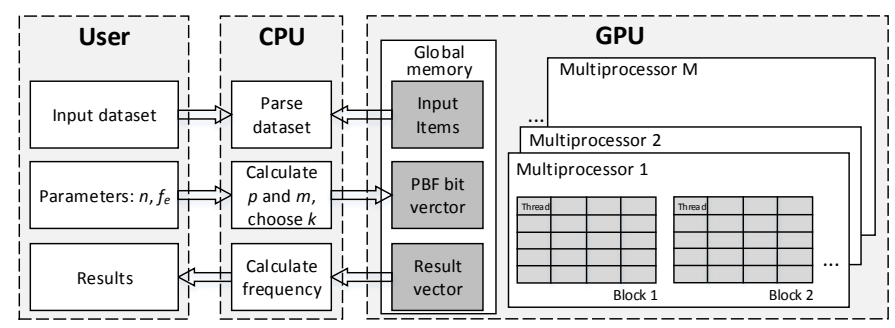

Figure 3: Design overview.

The host program initializes the PBF's parameters and calculates frequency results based on the result vector, which contains the number of $1 \mathrm{~s}$ of each queried item returned by GPU. Furthermore, the major function of the host program is to parse the input dataset, since the GPU is not able to handle data I/O. Specifically, our approach works as follows: first, we adapt the approach proposed in [33], where we concatenate all input flows to one single, long flow. In order to access each flow, we keep record of the starting addresses of each flow in an index table. Second, since duplicate queries for the same flow always return the same frequency result, we implement a classic Bloom filter to eliminate duplicate query inputs as an integrated part of the PBF design. Note that this assisting structure consumes much less storage compared to the main data structure. In other words, in the QUERY operation, the original queries will first pass a classic Bloom filter, and only those unique flows will be transferred to GPU's memory for processing. This saves considerably memory on the GPU side as many redundant queries will be eliminated. Third, due to the relatively smaller memory size of GPUs compared to CPUs, the GPU's memory is usually not sufficient to store all the data, which includes the input flows, the PBF bit vector, and the result vector in the QUERY operation. In such scenarios, the host will partition the input dataset into smaller batches, which are then transferred to the GPU side sequentially. After processing, the GPU will return the results in batches as well. In the end, the host will combine results of all batches to yield the final result.

\subsection{Parallel Operations of the PBF}

We then describe how to implement the two operations of the PBF on GPUs. In the INSERT operation, there are two levels of parallelism: we need to process multiple 


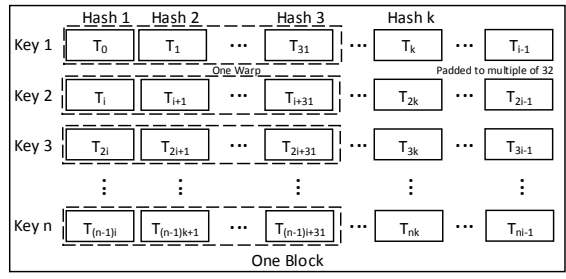

Figure 4: Block design option 1.

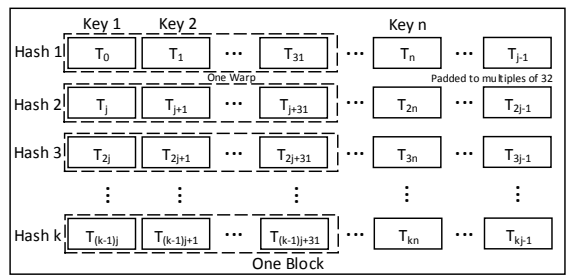

Figure 5: Block design option 2.

traffic flows, and for each input flow, we calculate multiple hash functions. Therefore, we first assign the GPU's blocks to be a two-dimensional matrix of threads. Next we have two design choices: first, each row of threads can calculate different hash functions and each column of threads can access different traffic flows, as shown in Figure 4. Alternatively, each row of threads can access different traffic flows and each column of threads can calculate different hash functions, as shown in Figure 5

We choose the first design in our PBF's implementation, and the reasons are as follows. Recall the two principles we presented to fully utilize GPU's computation power: in the first design, all threads in one warp will access the same flow, which gets broadcast with high time efficiency. On the other hand, in the second design, all threads in one warp will calculate hash functions of different traffic flows, which might have different lengths. The hash function we choose is murmurhash [38], whose computation overhead is proportional to the length of the input string. Hence, for this design, there will be unbalanced computation tasks across threads in one warp, a problem that makes the second design to be less efficient compared with the first one.

Based on this design choice, specifically, we utilize a matrix of threads in each block. The number of columns is assigned to be the number of hash functions padded to nearest multiple of the warp size, i.e., 32 . The reason of padding is to make sure all 
threads in one warp will access the same flow. The number of rows can be calculated as the maximum number of threads in one block divided by the number of columns. In order to utilize all the threads, the number of flows processed in one block is set to be a multiple of the number of rows. For instance, if the number of hash functions is 200 and the maximum number of threads in one block is 1024 , the thread block dimension should be $224 \times 4$, hence there are 896 threads in each block, and the number of flows processed in one block could be $\{4,8,12, \ldots\}$. Based on this design, Algorithm 6 describes the INSERT operation of the PBF on GPUs.

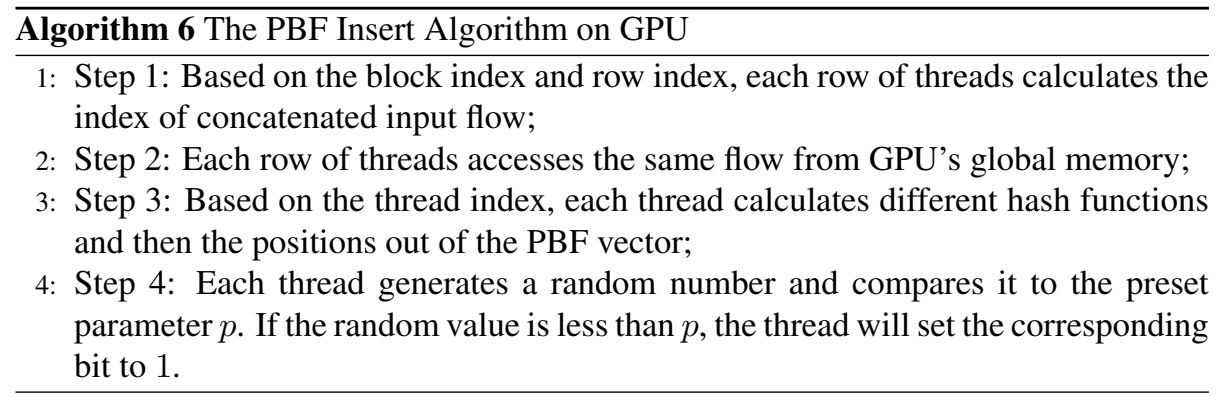

In the QUERY operation, according to Algorithm 2, we need to count how many bits have been set to 1 s out of $k$ hashed positions. If we use the same thread dimension assignment as in the INSERT operation, we need to use atomic operations to add the result across different threads. In most cases, the atomic operation on GPUs are too costly for our needs. Therefore, in the QUERY operation, we make sure that only one thread will calculate all the hash functions for one flow, as opposed to allowing different threads to share hashing tasks for one flow (as in the INSERT algorithm). The QUERY operation is shown in Algorithm 7 .

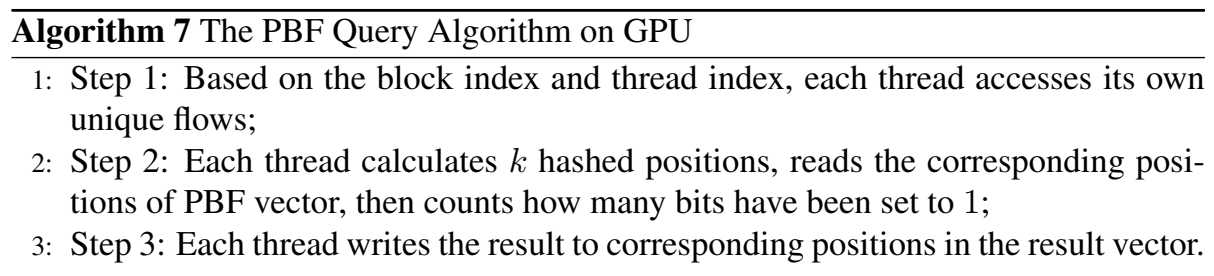




\section{Evaluation}

In this section, we compare the performance of PBF with following baselines, one non-Bloom-filter based approach: count-min sketch algorithm [22], and two Bloom-

\subsection{Flow frequency estimation}

We utilize the first five-minute duration traffic dataset to compare performance of the PBF with one non-Bloom-filter based approach, count-min sketch [22]. We set the parameters such that two approaches use the same amount of memory, and then compare their frequency estimation accuracy. For count-min sketch, we set $\epsilon=0.0001, \delta=$ 


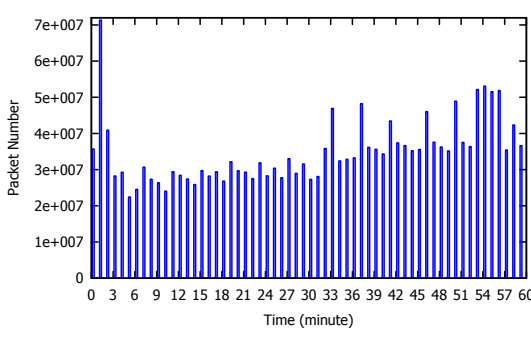

Figure 6: Dataset traffic pattern, generated based on 39

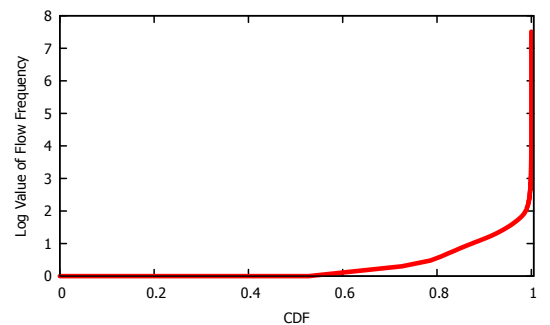

Figure 7: Dataset flow frequency, generated based on 39
455 a 32 bit counter, the number of bits that count-min sketch needs is $10,438,272$. In terms of the PBF, we set $m=10,438,272, p=0.0001$. For better comparison, we plot the sorted relative errors. In Figure 8, we plot the relative errors of all flows. In Figure 9. Figure 10, and Figure 11, we set the frequency threshold as 100,500 and 1000 accordingly, and only plot results of those flows whose frequency exceed the threshold. Comparing four figures, PBF performs better in terms of all flows and lower frequency threshold (100 and 500), while count-min sketch algorithm performs better when frequency is over 1000 .

Next, we evaluate frequency estimation accuracy of PBF on frequent flows, comparing with two Bloom-filter based approaches: counting Bloom Filter (CBF) [25], and multi-resolution space-code Bloom filter (MRSCBF) [29]. For PBF parameters, we set $p=0.00005, k=4,000$, and $m=800 M$. The comparison between the real frequency and the estimated frequency of the first 500 frequent flows $\left(f_{\text {thre }}=1000\right.$, sorted by time) are shown in Figure 12 . Observe that the estimated frequency and the real frequency are almost perfect matching each other. The only exceptions are those cases when the PBF loses its estimation ability as it's $k$ hash bits are saturated with $1 \mathrm{~s}$, leading it to miss certain data points. However, we argue that such missing points have no effects on the identification of heavy hitters, as these missing points correspond to heavy flows with a high certainty.

Next, we compare with the baselines, the CBF and the MRSCBF. For the CBF method, we set $k=4000$ and $m=800 M$. However, to prevent counter overflows, 


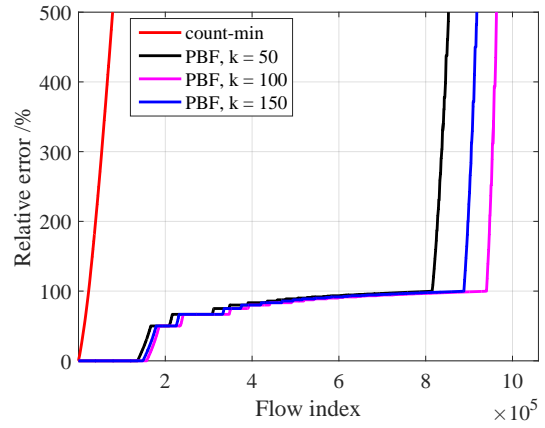

Figure 8: Relative error of estimated frequency compared with count-min.

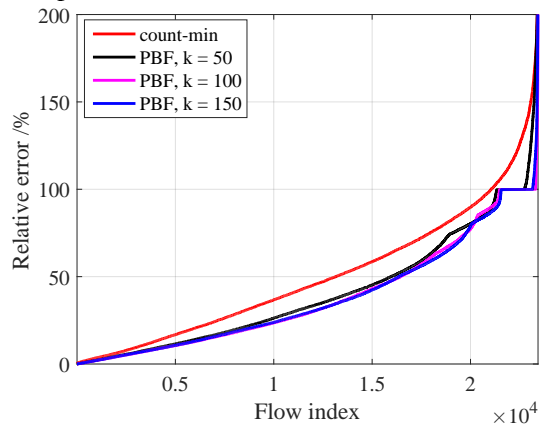

Figure 10: Relative error of estimated frequency compared with count-min, $f_{\text {thre }}=500$.

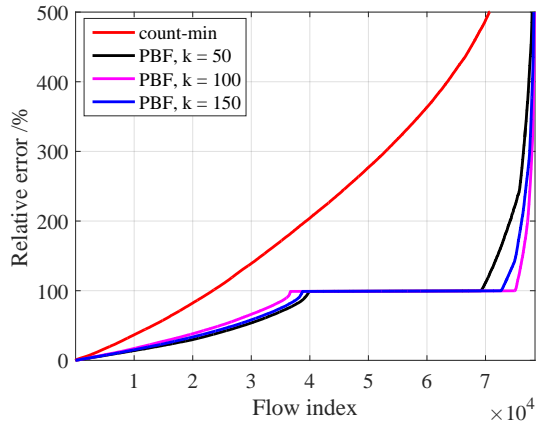

Figure 9: Relative error of estimated frequency compared with count-min, $f_{\text {thre }}=100$.

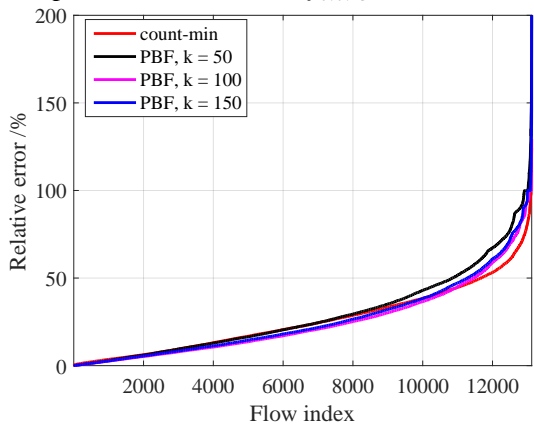

Figure 11: Relative error of estimated frequency compared with count-min, $f_{\text {thre }}=1000$.

we have to set each counter to occupy 22 bits, which means that in terms of memory overhead, the CBF takes 22 times of the memory compared to PBF to deliver accurate counting performance. For the second baseline MRSCBF method, we set $l=32$, $r=6$, and $k=\{3,4,6,6,6,6\}$ to accommodate the most frequent flows. To reduce the computation time of the look-up table, we set the maximum estimated frequency as 10, 000, which has no effects on detecting heavy hitter flows. This is why the estimated frequency is never larger than 10,000. Excluding the flows with frequencies more than 10,000 , the average estimation error of MRSCBF is $9.3 \%$, which is more than twice of the error of PBF. On the other hand, it needs $\sum_{i=1}^{6} m_{i}=22,117,154,656$ bits of memory to hold this MRSCBF, which means that the MRSCBF takes 27.6 times of the memory compared to PBF. All the comparison results in terms of average relative errors and memory usage could also be inferred in Table 6 


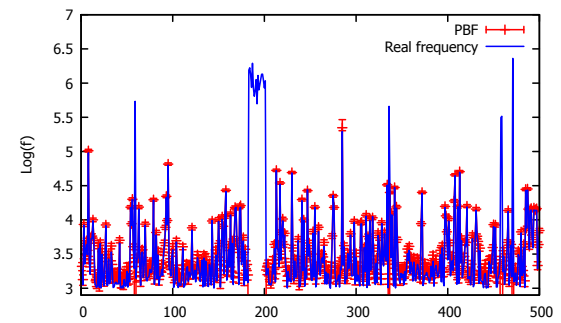

Figure 12: Comparison between the real frequency and the estimated frequency with the PBF.

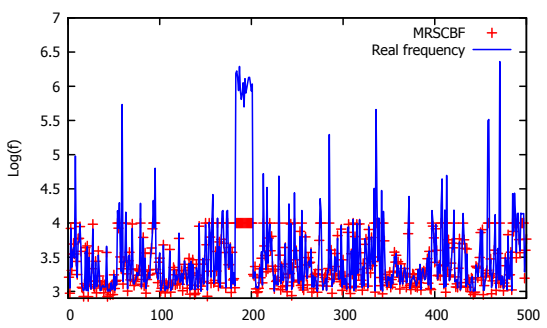

Figure 14: Comparison between the real frequency and the estimated frequency with the MRSCBF.

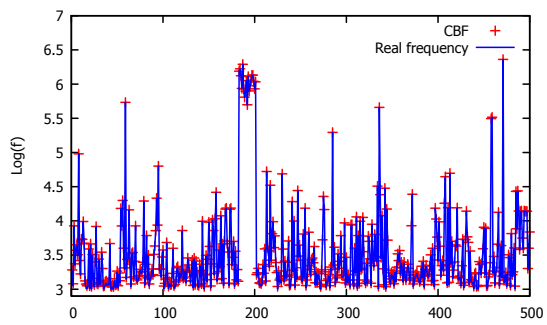

Figure 13: Comparison between the real frequency and the estimated frequency with the CBF.

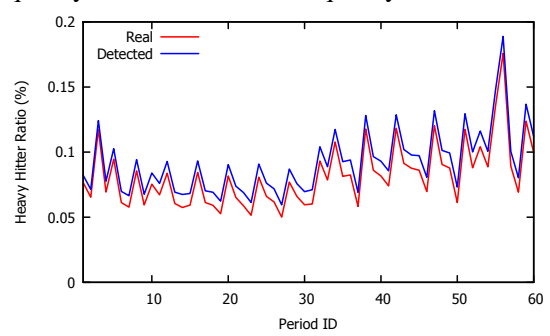

Figure 15: Comparison of the real heavy hitter ratio and the detected heavy hitter ratio of the TPBF.

Table 6: Comparison with Baselines

\begin{tabular}{c|c|c}
\hline Approaches & Average Relative Error & Memory Usage \\
\hline PBF & $3.83 \%$ & $100 \mathrm{MB}$ \\
\hline CBF & $3.76 \%$ & $2200 \mathrm{MB}$ \\
\hline MRSCBF & $9.3 \%$ & $2765 \mathrm{MB}$ \\
\hline
\end{tabular}

\subsection{Long-term Heavy Flow Detection with T-PBF}

using a dataset which constains an hour durace traffic traces. For the T-PBF, we set $p=0.00005, k=4000, m=800 M$, and $q=0.3$. The decay operation is triggered after one minute's traffic data. We calculated the detected heavy hitter ratio (the number of detected heavy flows over the total number of flows), the false-positive ratio, and the false-negative ratio for each period. For both the false-positive ratio and the falsenegative ratio, the threshold on frequency is set as 1000. As shown in Figure 15, the detected heavy hitter ratio on average is $6.48 \%$ larger than the real heavy hitter ratio. The source of this inaccuracy comes from the accumulation of flow frequencies in the 
previous periods. In general, the T-PBF works as expected, as we can observe that its small estimation errors and low memory overhead.

\subsection{Compare CPU and GPU implementations}

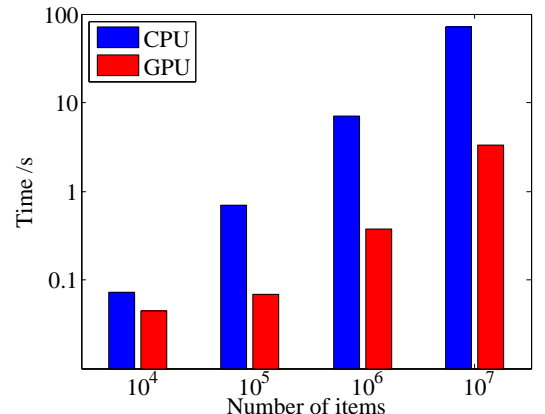

Figure 16: Run time comparison as the number of items varies.

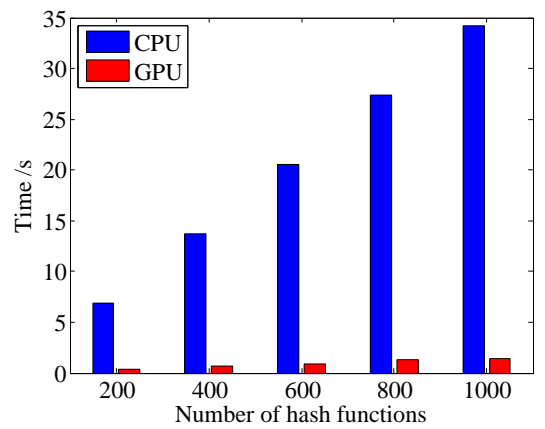

Figure 18: Run time comparison as the number of hash functions varies.

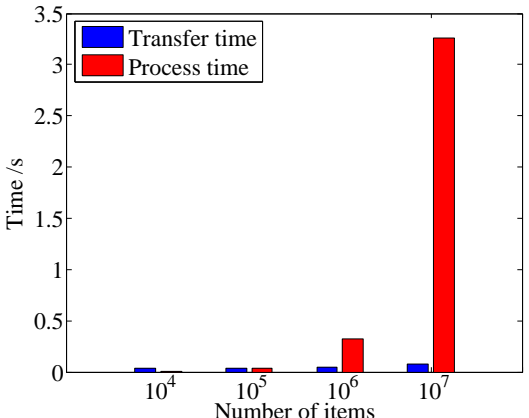

Figure 17: Comparison of transfer and processing time on GPU.

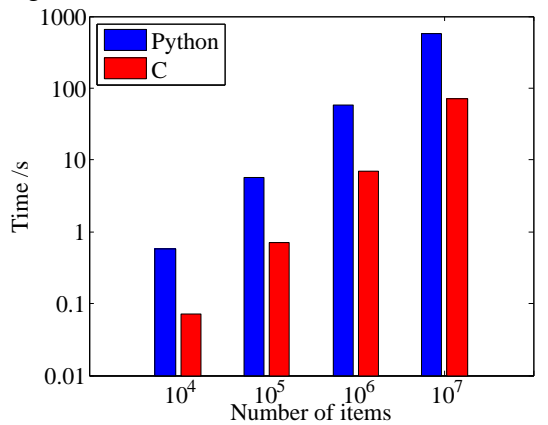

Figure 19: Run time comparison between python package and C code.

We next evaluate the PBF's implementation on GPUs. We generate random strings as workloads, whose frequencies follow Zipf's law distribution with the parameter $s=$ 0.1. For hardware, We choose the GPU model as NIVIDIA GT640 with 384 CUDA cores and 1GB global memory, and the CPU model as Intel i7 at 3.4GHz. Both the GPU and the CPU are main stream models. The GPU is installed with CUDA 6.5 toolkit. For each dataset and each set of parameters, we conduct 5 times of experiments, and calculate the average value as final run time.

First, to verify the correctness of PBF's implementation on GPU, we use the same input dataset, as well as the same parameter settings on both CPU and GPU implemen- 
tations. Therefore, all the operations regarding to the PBF vector are the same except that the GPU implementation runs in a parallel manner. Through comparison, the frequency results are the same of GPU and CPU implementations, which demonstrates that our GPU implementation is indeed correct.

Next we compare the runtime of GPU and CPU implementations. We consider total number of input items as configuration parameters, which we set to be $\left\{10^{4}, 10^{5}, 10^{6}, 10^{7}\right\}$, respectively. According to Figure 16, we can calculate the processing throughput (in terms of processed items per second) for GPU implementation is 200, 000, 1, 250, 000, $3,125,000$ and 3, 125, 000, accordingly. Furthermore, PBF implementation on GPU is about 10 times faster than the corresponding one on the CPU. Observe that the processing time on the CPU side increases linearly as the number of items increase, which verifies that the time processing each item is constant. However, the processing time (as well as processing rate) on GPU is not perfectly linear. The reason is that the data transfer between CPU and GPU takes additional, non-linear time. As shown in Figure 17. when the dataset size is small, the transfer time is comparable to, or even larger than the processing time. However, as the dataset size increases, the transfer time only takes small portion of the whole processing time, which results in much better performance for the GPU side. Therefore, The PBF implemented on GPU is preferred for processing large amount of data.

Finally, we also plot the runtime using different number of hash functions in Figure 18. We observe the similar results: as the number of hash function increases, we encounter more speedup since we could achieve more parallelism using GPUs.

\subsection{Python package verification}

To facilitate other researchers to adopt our design, we have released an open-source package for the PBF in Python, and this package has been indexed by the official Python website [9]. We next compare the performance of our developed python package to the original $\mathrm{C}$ code, and plot the run time comparison in Figure 19, with different number of input items. As shown in the figure, the python package is around 8 times slower than the $\mathrm{C}$ code implementation, which is common due to the interpreting nature of python packages. 


\section{Conclusion}

In this paper, we develop the probabilistic Bloom filter (PBF), which extends conventional Bloom filters to perform probabilistic counting operations. We provide the PBF's APIs to demonstrate how they can be used by applications, and quantitatively investigate the performance of the PBF through analytical approaches. The derived closed-form results answer our questions regarding the capacity, accuracy, and parameter selection of the PBF. In addition, we also extend the PBF into a time-decaying PBF (T-PBF) for dynamic data applications. Finally, we implement the PBF in GPUs to gain more time efficiency. Our evaluation results based on realistic network dataset show that this design outperforms the existing, state-of-the-art approaches.

To the best of our knowledge, our work in this paper is the first probabilistic Bloom filter that is designed to count large volume of data with adjustable capacity and accuracy. We hope our work can stimulate future work in this direction, and provide a basis for investigations towards better methods based on probabilistic counting and Bloom filters.

\section{Acknowledgement}

This work was supported by the National Science Foundation under Grant CNS1239478 and Grant CNS-0953238.

\section{References}

[1] N. Sarrar, S. Uhlig, A. Feldmann, R. Sherwood, X. Huang, Leveraging Zipf S Law For Traffic Offloading, ACM SIGCOMM Computer Communication Review 42 (1) (2012) 16-22.

[2] T. Li, S. Chen, Y. Ling, Per-Flow Traffic Measurement Through Randomized Counter Sharing, IEEE/ACM Transactions on Networking 20 (5) (2012) 16221634. 
[3] C. Estan, G. Varghese, New Directions in Traffic Measurement and Accounting, in: Proceedings of the ACM SIGCOMM Conference on Applications, Technologies, Architectures, and Protocols for Computer Communications, Vol. 32, 2002, p. 75.

[4] D. Hoplaros, Z. Tari, I. Khalil, Data summarization for network traffic monitoring, Journal of Network and Computer Applications 37 (2014) $194-205$.

[5] C. Estan, G. Varghese, M. Fisk, Bitmap Algorithms for Counting Active Flows on High-Speed Links, IEEE/ACM Transactions on Networking 14 (5) (2006) 925937.

575 [6] Cisco, Cisco IOS NetFlow, http://http://www.cisco.com/c/en/ us/products/ios-nx-os-software/ios-netflow/index.html [Online; accessed October 10, 2014] (2012).

[7] Y. Yao, S. Xiong, J. Liao, M. Berry, H. Qi, Q. Cao, Identifying frequent flows in large datasets through probabilistic bloom filters, in: Quality of Service, 2015. IWQoS 2015. IEEE/ACM International Symposium on, 2015.

[8] F. Khan, N. Hosein, S. Ghiasi, C. N. Chuah, P. Sharma, Streaming solutions for fine-grained network traffic measurements and analysis, IEEE/ACM Transactions on Networking 22 (2) (2014) 377-390.

[9] Python package for the PBF, http://github.com/xsswfm/ pypbf/https://pypi.python.org/pypi/pypbf/0.0.0, [Online; accessed August 5,2015] (2015).

[10] N. Manerikar, T. Palpanas, Frequent items in streaming data: An experimental evaluation of the state-of-the-art, Data and Knowledge Engineering 68 (4) (2009) $415-430$.

[11] G. Cormode, M. Hadjieleftheriou, Finding frequent items in data streams, Proc. VLDB Endow. 1 (2) (2008) 1530-1541. 
[12] S. Gangam, P. Sharma, S. Fahmy, Pegasus: Precision hunting for icebergs and anomalies in network flows, in: 2013 Proceedings IEEE INFOCOM, 2013, pp. $1420-1428$.

[13] C. Estan, G. Varghese, New directions in traffic measurement and accounting: Focusing on the elephants, ignoring the mice, ACM Trans. Comput. Syst. 21 (3) (2003) 270-313.

[14] B. Whitehead, C.-H. Lung, P. Rabinovitch, An efficient hybrid approach to perflow state tracking for high-speed networks, Computer Communications 36 (8) (2013) $927-938$.

[15] Y. Li, H. Wu, T. Pan, H. Dai, J. Lu, B. Liu, Case: Cache-assisted stretchable estimator for high speed per-flow measurement, in: IEEE INFOCOM 2016 The 35th Annual IEEE International Conference on Computer Communications, 2016, pp. 1-9.

[16] E. D. Demaine, A. López-Ortiz, J. I. Munro, Frequency Estimation of Internet Packet Streams with Limited Space, Springer Berlin Heidelberg, Berlin, Heidelberg, 2002, pp. 348-360.

[17] R. M. Karp, S. Shenker, C. H. Papadimitriou, A simple algorithm for finding frequent elements in streams and bags, ACM Trans. Database Syst. 28 (1) (2003) $51-55$.

[18] G. S. Manku, R. Motwani, Approximate frequency counts over data streams, in: Proceedings of the 28th International Conference on Very Large Data Bases, VLDB '02, VLDB Endowment, 2002, pp. 346-357.

[19] A. Metwally, D. Agrawal, A. El Abbadi, Efficient Computation of Frequent and Top-k Elements in Data Streams, Springer Berlin Heidelberg, Berlin, Heidelberg, 2005, pp. 398-412.

[20] M. Charikar, K. Chen, M. Farach-Colton, Finding Frequent Items in Data Streams, Springer Berlin Heidelberg, Berlin, Heidelberg, 2002, pp. 693-703. 
[21] G. Cormode, S. Muthukrishnan, An improved data stream summary: the countmin sketch and its applications, Journal of Algorithms 55 (1) (2005) 58 - 75.

[22] G. Cormode, S. Muthukrishnan, What's new: Finding significant differences in network data streams, IEEE/ACM Trans. Netw. 13 (6) (2005) 1219-1232.

[23] C. Jin, W. Qian, C. Sha, J. X. Yu, A. Zhou, Dynamically maintaining frequent items over a data stream, in: Proceedings of the Twelfth International Conference on Information and Knowledge Management, CIKM '03, ACM, New York, NY, USA, 2003, pp. 287-294.

[24] B. H. Bloom, Space/time trade-offs in hash coding with allowable errors, Commun. ACM 13 (7) (1970) 422-426.

[25] L. Fan, P. Cao, J. Almeida, A. Z. Broder, Summary cache: a scalable wide-area web cache sharing protocol, IEEE/ACM Trans. Netw. 8 (3) (2000) 281-293.

[26] A. Broder, M. Mitzenmacher, Network Applications of Bloom Filters: A Survey, Internet Mathematics 1 (4) (2003) 485-509.

[27] S. Tarkoma, C. E. Rothenberg, E. Lagerspetz, Theory and Practice of Bloom Filters for Distributed Systems, IEEE Communications Surveys and Tutorials 14 (1) (2012) 131-155.

[28] K. Cheng, L. Xiang, M. Iwaihara, Time-decaying bloom filters for data streams with skewed distributions, in: 15th International Workshop on Research Issues in Data Engineering: Stream Data Mining and Applications (RIDE-SDMA'05), 2005, pp. 63-69.

[29] A. Kumar, J. Xu, J. Wang, Space-code bloom filter for efficient per-flow traffic measurement, IEEE J.Sel. A. Commun. 24 (12) (2006) 2327-2339.

[30] W. Liu, W. Qu, Z. Liu, K. Li, J. Gong, Identifying elephant flows using a reversible multilayer hashed counting bloom filter, in: High Performance Computing and Communication 2012 IEEE 9th International Conference on Embedded 
Software and Systems (HPCC-ICESS), 2012 IEEE 14th International Conference on, 2012, pp. 246-253.

[31] L. Bai, J. Wu, Elephant Flows Identification Based on Dynamic Multiple Filters Algorithm, Springer London, London, 2013, pp. 797-804.

[32] Z. Zhang, B. Wang, J. Lan, Identifying elephant flows in internet backbone traffic with bloom filters and $\{$ LRU $\}$, Computer Communications 61 (2015) $70-78$.

[33] L. Costa, S. Al-Kiswany, M. Ripeanu, Gpu support for batch oriented workloads, in: Performance Computing and Communications Conference (IPCCC), 2009 IEEE 28th International, 2009, pp. 231-238.

[34] H. Shi, B. Schmidt, W. Liu, W. Muller Wittig, A parallel algorithm for error correction in high-throughput short-read data on cuda-enabled graphics hardware, Journal of Computational Biology 17 (4) (2010) 603-615.

[35] L. Ma, R. Chamberlain, J. Buhler, M. Franklin, Bloom filter performance on graphics engines, in: Parallel Processing (ICPP), 2011 International Conference on, 2011, pp. 522-531.

[36] G. Robert, A primer in game theory, FT Prentice Hall Publisher, London.

[37] NVIDIA CUDA Programming Guide, http://docs.nvidia.com/cuda/ cuda-c-programming-guide/, [Online; accessed June 20, 2015] (2013).

[38] Murmurhash, https://sites.google.com/site/murmurhash/, [Online; accessed July 18, 2015] (2011).

[39] Internet trace data from caida [Online, accessed September 9, 2014] (2013).

URL https://data.caida.org/datasets/passive-2013/ 